\title{
Dark matter versus magnetism
}

\section{James Binney}

TIIE strongest evidence for the existence of dark matter in the Universe comes from the rotation velocity of gas orbiting in spiral galaxies: this does not fall off with increasing galactocentric radius as quickly as one would expect from the visible distribution of mass. Elsewhere in this issue (Nature 360, 652-653; 1992), E. Battaner et al. suggest that this evidence may be flawed because it fails to take into account the effects of magnetic stresses within the observed gas.

Interstellar gas generally contains sufficient free electrons to act as a near perfect conductor, able to sustain the currents to support a magnetic field. Also, from the alignment of dust grains, a few measurements of Zeeman splittings of spectral lines, the observation of synchrotron emission by relativistic electrons and from the observation of Faraday rotation of polarized radio emission, we know that interstellar gas is permeated by a magnetic field. A field of 1 picotesla (10 microgauss) or more would exert forces on the gas sufficient to affect its dynamics significantly.

Back in the 1950 s it was widely believed that spiral structure in galaxies was a predominantly magnetic phenomenon, as were the striking intergalactic filaments seen near strange galaxies such as NGC4038/9. But from the mid-

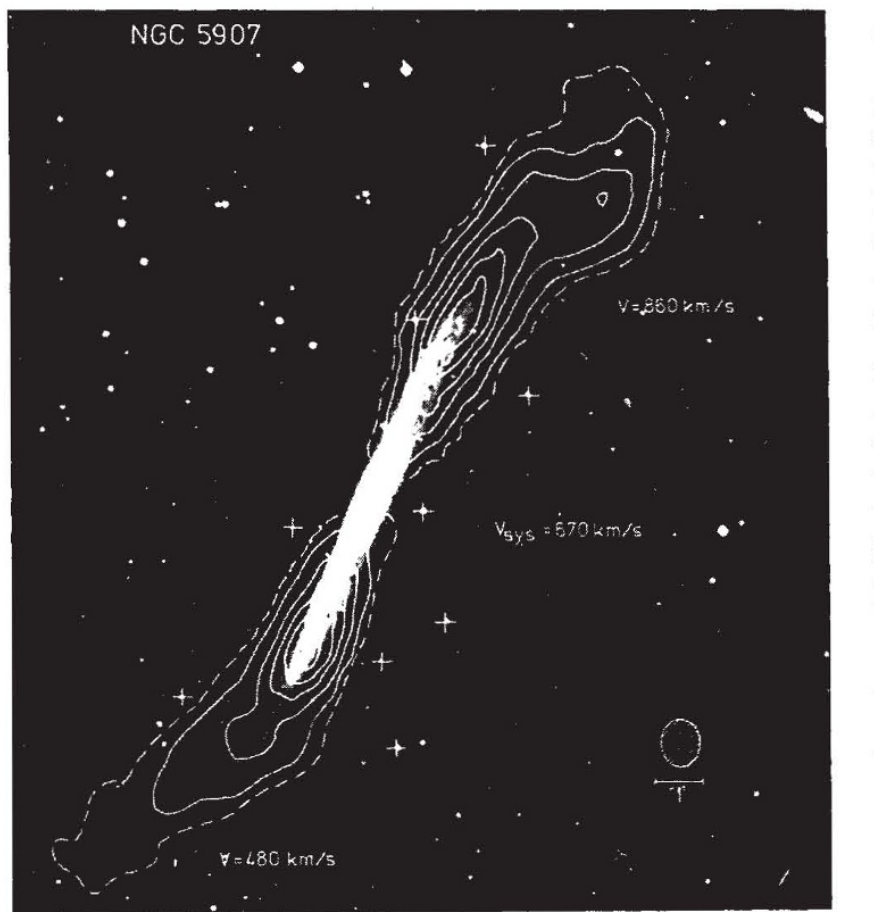

Optical image of the stellar disk of the galaxy NGC5907, with radio-emission contours showing hydrogen flaring at the edges a sign of magnetic tension?

1960 s onwards it became increasingly clear that gravity acting alone could account for spiral structure; and intergalactic filaments turned out to arise naturally when a disk galaxy is tidally distorted by a close companion (J. Barnes and L. Hernquist, A. Rev. Astr. Astrophys. 30, 705-742; 1992). So the conventional position nowadays is that although the interstellar magnetic field probably plays a key role on parsec scales and less, on galactic scales it amounts to no more than a small perturbation on fundamentally gravitational dynamics.

Battaner et al. challenge this consensus by arguing that tens of kiloparsecs from the centre of a galaxy, where the gas density is usually very low, the dynamics of the gas may be strongly affected by a magnetic field of the order of 1 picotesla. We know that the interstellar field tends to wind around galactic disks, so that to a first approximation one can imagine that the field lines form
The magnetic tension in the hoop must be counteracted by some combination of a net outward force on the hoop due to a radial gradient in the perpendicular magnetic pressure, and an excess of the centrifugal force on the spinning hoop over the inward gravitational pull of the galaxy. Battaner et al. deduce the field configuration within the Andromeda galaxy, M31, that would cause the gas to rotate at the observed speeds at each radius if the only matter in the galaxy were that associated with the observed stars. They find that a field of magnitude 0.8 picotesla from 10 to 30 kiloparsecs does the trick.

How might one confirm that a field of this magnitude is present? From the alignment of dust grains one learns the orientation but not the magnitude of the field, and the field is much too weak to be detected from a Zeeman splitting. This leaves Faraday rotation and synchrotron emission as possible probes.

a series of gas-filled hoops within the plane of the disk. The magnetic field $B$ imposes two kinds of forces on the hoop: a tension $1 / 2 B^{2} / \mu_{0}$ runs the long way around the hoop, pulling it more snugly around the galaxy, while in the two perpendicular directions, the field exerts pressure $1 / 2 B^{2} / \mu_{0}$, tending to cause the hoop to fatten into a doughnut (where $\mu_{0}$ is the permittivity of free space). the product of the energy densities $B^{2} / 2 \mu_{0}$ and $\varepsilon$ in the field and in relativistic electrons. Battaner et al. obtain a reasonable fit to the synchrotron radiation observed at large radii from M31 by assuming that $\varepsilon$ scales with the gas density $\rho$ as $\rho / B$; the motivation for this assumption is that the source of the relativistic electrons is the gas, and synchrotron losses due to $B$ constitute the sink. But this calculation does not form a rigorous test of the theory in that the normalization of the density of relativistic electrons is set by requiring that theory and observation agree at 10 kiloparsecs.

In our own Galaxy, Faraday rotation of radiation from pulsars has been used to probe the magnetic field along many lines of sight. The conclusion of these studies (L. Spitzer Physical Processes in the Interstellar Medium; Wiley, New York, 1978) is that near the Sun the azimuthal field is around 0.2 picotesla, a factor of four smaller than the field required by Battaner et al. in the outer regions of M31. As magnetic forces are proportional to $B^{2}$, these forces are locally more than an order of magnitude smaller than those required by Battaner et al.

Unfortunately, in regions significantly further from the Galactic Centre than we are, the azimuthal field makes only a small contribution to measurements of Faraday rotation, because it runs almost perpendicular to the relevant lines of sight. Such measurements, therefore, can probably

not rule out the possibility of an outwardly increasing azimuthal field of the type required by Battaner et al. But studies of the vertical structure of the interstellar medium should allow one to place a firm upper limit on the dynamical significance of the azimuthal field. Indeed, if the azimuthal tension associated with $B$ is strong enough to make a serious contribution to the centripetal force, the perpendicular pressure will prevent the gas disk being strongly flattened. Hence, as one moves out into the region of dynamically significant field, the gas disk should flare very strongly. Observations of other galaxies show that galactic disks do flare at the edge of the galaxies' optical images (see figure). But do they flare as rapidly as the model of Battaner et al. requires?

James Binney is in the Department of Physics, Oxford University, 1 Keble Road, Oxford OX1 3NP, UK. 